patients $(68.2 \%)$ continued antimalarials after introduction. Retinal alterations were not associated with age, disease length and duration of the antimalarial therapy.

Conclusions We observed a similar frequency of antimalarial suspension as reported in other studies. The main adverse events during the therapy were ocular toxicity, but in a percentage of patients remains the main cause of treatment withdrawal.

\section{P168 PREVALENCE AND CHARACTERISTICS OF CARDIOVASCULAR AUTONOMIC DYSFUNCTION IN PATIENTS WITH SYSTEMIC LUPUS ERYTHEMATOSUS}

${ }^{1}$ Amanda Zinglersen, ${ }^{1}$ Katrine Iversen, ${ }^{2}$ Esben Laugesen, ${ }^{3}$ Jesper Fleischer, ${ }^{1}$ Søren Jacobsen. ${ }^{1}$ Copenhagen Lupus and Vasculitis Clinic, Center for Rheumatology and Spine Diseases, Rigshospitalet, Copenhagen; ${ }^{2}$ Dept. of Endocrinology and Internal Medicine, Aarhus University Hospital, Aarhus; ${ }^{3}$ Steno Diabetes Center Aarhus, Aarhus University Hospital, Aarhus, Denmark

\subsection{6/lupus-2020-eurolupus. 210}

Background The prevalence of cardiovascular autonomic dysfunction (CAN) has previously been described with large variation (10-90\%) in patients with systemic lupus erythematosus (SLE). CAN is assessed by heart rate variability (HRV) or cardiovascular autonomic reflex tests (CARTs), but these two methods have not previously been compared in SLE patients. Further, little is known about the autonomic nervous system (ANS) impairments at different stages of CAN. Consequently, the purpose of this study is, in a large cohort of SLE patients, to determine the prevalence of CAN and to characterize the ANS function at different CAN-stages by HRV and CARTs.

Methods CAN was tested in 111 SLE patients with a 5-minute HRV-test and three CARTs. HRV-items reflecting parasympathetic (PNS) function (high frequency power, HFP) and mixed PNS-sympathetic (SNS) function (low frequency power, LFP; total power, TP; LFP/HFP-ratio and peak LF) were calculated. CAN was staged by the number of abnormal CARTs; early CAN: one abnormal CART, definite CAN: two or more abnormal CARTs. Fifty-five SLE patients were age and gender matched to 55 CAN-tested healthy controls (HC).

Results The prevalence of definite CAN in SLE is higher than in HCs $(24.1 \%$ vs. $1.9 \%, p=0.001)$. CAN-stage was significantly associated all HRV-measures, except LF/HF-ratio. SLE patients without definite CAN had lower PNS activity than HCs without definite CAN (HFP: 42.7 vs. $87.5 \mathrm{~ms}^{2}$, $p=0.006$; LFP/HFP-ratio: 1.79 vs. $1.00, p=0.010$, resp.). Furthermore, SLE patients with definite CAN had signs of lower mixed PNS-SNS-function as determined by LFP, TP and peak LF (all $\mathrm{p}<0.05$ ), see table 1 .
Conclusions The prevalence of CAN in SLE patients was 12 times higher than in HCs. In SLE patients, early CAN was associated with impaired PNS function, whereas definite CAN was characterized by impaired function of the PNS and SNS.

\section{P169 ASSOCIATION BETWEEN CARDIOVASCULAR AUTONOMIC NEUROPATHY AND QUALITY OF LIFE IN PATIENTS WITH SYSTEMIC LUPUS ERYTHEMATOSUS}

${ }^{1}$ Amanda Zinglersen, ${ }^{1}$ Henrik Leffers, ${ }^{1}$ Katrine Iversen, ${ }^{2}$ Jesper Fleischer, ${ }^{1}$ Søren Jacobsen. ${ }^{1}$ Copenhagen Lupus and Vasculitis Clinic, Center for Rheumatology and Spine Diseases, Rigshospitalet, Copenhagen; ${ }^{2}$ Steno Diabetes Center Aarhus, Aarhus University Hospital, Aarhus, Denmark

\subsection{6/lupus-2020-eurolupus.211}

Background Cardiovascular autonomic neuropathy (CAN) with comprised function of the parasympathetic (PNS) and/or the sympathetic nervous system (SNS) occurs frequently (10$90 \%$ ) in patients with systemic lupus erythematosus (SLE). CAN is a subclinical dysfunction which is reflected by reduced heart rate variability (HRV). Only a few studies have investigated the clinical impacts of CAN in SLE. However, impaired quality of life occurs frequently among SLE patients, and has, in other patient groups and in healthy individuals, been associated to impaired HRV. Consequently, the purpose of this study is to explore if CAN is associated to self-report of low health related quality of life (HRQoL) in SLE patients.

Methods CAN was tested in 87 SLE patients with a 5-minute HRV-test. HRV-measures reflecting PNS function (high frequency power, HFP) and mixed PNS-SNS function (low frequency power, LFP; total power (TP); low- vs high frequency power-ratio, LFP/HFP-ratio and peak LF frequency) were calculated. The patients were further asked to complete a questionnaire on severity of pain, depression and fatigue, adapted from the Systemic Lupus Activity Questionnaire (SLAQ), along with the questionnaire Short Form 12 (SF-12) on physical and mental HRQoL.

Results TP and peak LF were associated to low quality of life based on the physical domain of the SF-12 (TP: $\beta=0.247$, $p=0.025$ and peak LF: $\beta=0.225, p=0.037)$. However, no associations were observed between $\mathrm{AD}$ and pain, depression, fatigue or the mental domain from the SF-12.

Conclusions Cardiovascular autonomic neuropathy of mixed PNS and SNS impairment was associated to self-report of low physical quality of life in SLE, but not to any mental domains of life quality. Further studies are needed to elaborate on causality between of autonomic dysfunction and the physical component of life quality in SLE patients.

Abstract P168 Table 1 Measures of heart rate variability (HRV) by cardiovascular autonomic neuropathy (CAN) (-CAN = no and early CAN $+\mathrm{CAN}=$ definitive CAN) for age and gender matched systemic lupus erythematosus (SLE) patients and healthy controls (HC)

\begin{tabular}{|c|c|c|c|c|c|c|c|c|}
\hline \multirow[b]{2}{*}{ HRV measures, median (IQR) } & \multicolumn{2}{|l|}{ HC } & \multicolumn{6}{|c|}{ SLE matched to HC } \\
\hline & \multicolumn{2}{|c|}{-CAN, $n=53$} & \multicolumn{2}{|c|}{-CAN, $n=41$} & \multicolumn{2}{|c|}{+ CAN, $n=13$} & \multirow{2}{*}{$\frac{p^{*}}{0.429}$} & \multirow{2}{*}{$\frac{p^{* *}}{0.015}$} \\
\hline LFP, $\mathrm{ms}^{\wedge} 2$ & 77.7 & $(49.3-195)$ & 69.1 & (39.8-159) & 36.4 & $(10.3-66.8)$ & & \\
\hline HFP, $\mathrm{ms}^{\wedge} 2$ & 87.5 & $(38.4-201)$ & 42.7 & $(18.2-76.2)$ & 5.30 & $(2.66-60.1)$ & 0.006 & 0.006 \\
\hline $\mathrm{TP}, \mathrm{ms}^{\wedge} 2$ & 378 & $(209-832)$ & 301 & $(151-495)$ & 115 & $(51.0-263)$ & 0.141 & 0.009 \\
\hline LFP/HFP-ratio & 1.00 & $(0.44-2.37)$ & 1.79 & $(1.00-3.50)$ & 2.94 & $(1.41-8.37)$ & 0.010 & 0.100 \\
\hline Peak LF, Hz & 0.04 & $(0.04-0.11)$ & 0.07 & $(0.04-0.12)$ & 0.04 & $(0.04-0.04)$ & 0.452 & 0.000 \\
\hline
\end{tabular}

